CAHIERS DE

NARRATOLOGIE

\section{Cahiers de Narratologie}

Analyse et théorie narratives

$21 \mid 2011$

Rencontres de narrativités : perspectives sur l'intrigue musicale

\title{
Musique et référentialité sur les titres des disques Blue Note
}

\section{Philippe Carrard}

\section{(2) OpenEdition}

Journals

Édition électronique

URL : http://journals.openedition.org/narratologie/6420

DOI : $10.4000 /$ narratologie. 6420

ISSN : 1765-307X

Éditeur

LIRCES

Référence électronique

Philippe Carrard, "Musique et référentialité sur les titres des disques Blue Note », Cahiers de

Narratologie [En ligne], 21 | 2011, mis en ligne le 20 décembre 2011, consulté le 19 avril 2019. URL:

http://journals.openedition.org/narratologie/6420; DOI : 10.4000/narratologie.6420

Ce document a été généré automatiquement le 19 avril 2019

Article L.111-1 du Code de la propriété intellectuelle. 


\title{
Musique et référentialité sur les titres des disques Blue Note
}

\author{
Philippe Carrard
}

1 Qu'ils soient catégorisés comme appartenant à la musique classique ou aux genres dits populaires, les disques sont en général placés dans une pochette qui donne quelques renseignements sur leur contenu. Cette information fait partie du paratexte (paradisque? ), ou plus précisément de ce que Gérard Genette appelle l'« épitexte éditorial » $(1987: 20)$ : la « zone incertaine » située entre le texte (le disque) et son contexte, zone où l'auditeur potentiel apprend qui joue, quoi, et quelle marque a procédé à l'enregistrement. Les pochettes que je me propose d'examiner sont celles de disques célèbres dans la communauté des amateurs de jazz: les albums enregistrés par le label Blue Note entre 1939 (date de la fondation de la compagnie par Alfred Lion et Francis Wolf) et 1965 (date de la vente de Blue Note à Liberty Records) (sur ces points, voir Cuscuna et Ruppli 1988). Ces pochettes sont renommées pour leur design, en particulier pour leurs photographies de musiciens, souvent prises par Lion lui-même. Je reviendrai plus tard sur la fonction de cette iconographie, mais mon objectif est ici d'étudier une autre composante des jaquettes Blue Note, à savoir leurs titres. Les disques de jazz, en effet, sont avant tout connus par leurs titres, Kind of Blue de Miles Davis ou A Love Supreme de John Coltrane désignant en général, dans les analyses des critiques ou les discussions des mélomanes, l'album dans son ensemble, et non le morceau qui porte ce nom. L'analyse des titres est donc importante pour une "poétique » du jazz, définie comme l'étude des règles, des codes et des conventions qui définissent non la musique de jazz elle-même, mais les textes qui la prennent pour objet. Le fait que la plupart des albums Blue Note dont il sera question ici aient été réédités sous forme de CD n'est pas pertinent pour mon propos, puisque la première page du livret que contient le boîtier, dans la majorité des cas, reproduit le recto de la pochette originale.

2 Les titres des disques de jazz soulèvent plusieurs problèmes, et je ne traiterai ici que quelques-uns d'entre eux. Ainsi, bien que la question soit importante d'un point de vue historique, je ne me demanderai pas - pour utiliser le jeu de mots proposé par Jacques 
Derrida (1981: 7) - qui à Blue Note avait le «titre » de décider des titres. De même, je n'analyserai pas la structure syntaxique des titres retenus dans mon corpus. Leo Hoek a effectué ce travail à propos des œuvres littéraires, et je considère comme acquis que ses analyses s'appliquent également aux pochettes de disque : la plupart des énoncés qui constituent des titres sont semi-grammaticaux ou, plus précisément, "sans verbe » et « elliptiques » (Hoek 1981 : 54). Laissant de côté les questions historiques et stylistiques, je considère les titres des disques Blue Note du point de vue des informations qu'ils fournissent. Je commencerai par établir une typologie en cherchant à déterminer ce que ces titres nous disent du contenu musical des albums dont ils établissent l'identité. Puis, dans un second temps, je me demanderai dans quelle mesure une étude de ces mêmes titres nous permet de revisiter une question maintes fois posée par les sémioticiens, les philosophes et les musicologues: celle de savoir, non si la musique "raconte une histoire ", comme Michael Toolan cherche à l'établir dans son article, mais, de manière plus générale, si cette musique possède une signification, et comment cette signification peut être déterminée.

3 Examinés du point de vue des informations qu'ils communiquent, les titres des albums Blue Note peuvent être distribués en trois catégories principales: celles des titres dénotatifs, connotatifs et indexicaux.

\section{Titres dénotatifs}

4 Ces titres fournissent des « informations exactes » sur le contenu d'un album et n'ont pas besoin d'être « interprétés » par l'auditeur potentiel (Hoek 1981:171), du moins pas par un auditeur compétent. Ils ne font en effet que lister un fait, ou une série de faits, considérés comme pertinents pour l'identification d'un disque de jazz. Ces faits peuvent comprendre :

\subsection{Le nom de l'artiste et du groupe qu'il dirige}

5 Sonny Rollins, Sonny Rollins; Tal Farlow, Tal Farlow Quartet; Hank Mobley, Hank Mobley and His All Stars. L'information peut être limitée au prénom de l'artiste et/ou inclure une évaluation, souvent sous la forme d'un adjectif : Andrew Hill, Andrew ; Bud Powell, Bud!; Fats Navarro, The Fabulous Fats Navarro; J.J. Johnson, The Eminent J.J. Johnson. Dans la mesure où ils répètent le nom de l'artiste qui figure déjà sur la pochette, ces titres répondent d'une manière redondante à la question qui est sans doute la plus centrale dans le cas d'un disque de jazz: Qui joue? Car le jazz, au contraire de la musique classique, met l'accent sur les interprètes et prête moins d'attention aux compositeurs. Dans mon corpus, seuls deux titres mentionnent les noms des compositeurs des morceaux qui constituent l'album: Horace Silver, Six Pieces of Silver et Jimmy Smith, Jimmy Smith Plays Fats Waller. Mais Silver et son groupe sont aussi les interprètes des Six Pieces, et l'album qui contient les thèmes de Waller est intrigant parce que ces thèmes ne semblent pas se prêter au style de Smith.

\subsection{Le genre de la musique}

6 Albert Ammons, Boogie-Woogie Classics; Ike Quebec, Heavy Soul ; Kenny Dorham, Afro-Cuban ; Art Hodes, Dixieland Jubilee ; George Lewis, Echoes of New-Orleans ; Charlie Rouse, Bossa 
Nova Bacchanal ; Grant Green, The Latin Bit. Ces titres peuvent confirmer ce que nous savons de l'artiste : Ammons est un spécialiste du style de piano boogie-woogie, et Lewis, du style New-Orleans tel qu'il a survécu dans les lieux où il est censé être né. Mais les titres peuvent aussi signaler que l'artiste fait quelque chose qui, pour lui, est inhabituel : Dorham ne figure pas au nombre des spécialistes du style Afro-Cubain; Rouse, au contraire de Stan Getz, ne s'était pas converti à la bossa nova; et Green jouait dans le style hard bop au moment de la sortie de The Latin Bit en 1962. En d'autres termes, des titres tels que Bossa Nova Bacchanal et The Latin Bit ne font pas que nous donner des renseignements sur le contenu de l'album. Ils programment une confrontation entre la musique et ses interprètes, du moins pour les auditeurs avisés qui peuvent discerner ce que des artistes comme Rouse et Green font ici d'atypique.

\subsection{Les circonstances qui ont entouré la production du disque}

7 Clifford Brown, Memorial Album (Brown était mort récemment); Kenny Burrell, Introducing Kenny Burrell (c'est là le premier album de Burrell pour Blue Note) ; Art Blakey, A Night at Birdland (ce disque a été enregistrée sur le vif au club Birdland) ; Freddie Redd, The Music from The Connection (cette musique a été composée pour la pièce de théâtre The Connection); Clifford Jordan et Don Gilmore, Blowing in from Chicago (les deux saxophonistes viennent du Southside de Chicago). Certaines de ces "circonstances" semblent plus mémorables que d'autres. Le nom du club où a été effectué l'enregistrement est ainsi fréquemment mentionné : Jutta Hipp, At the Hickory House ; Sonny Rollins, A Night at the Village Vanguard; Jimmy Smith, Groovin' at Small's Paradise; Donald Byrd, At the Half Note Café ; Stanley Turrentine, Up at Minton's. Ces titres exploitent les noms d'endroits connus et transportent l'auditeur dans des lieux dont il a sans doute entendu parler mais où il n'a pas eu l'occasion de se rendre, à moins qu'il n'habite New York. L'information « enregistré sur le vif » comporte aussi une promesse d'authenticité, fondée sur la conviction, très répandue auprès de certains amateurs, que le "meilleur » jazz se joue dans les clubs, et pas dans l'environnement d'un studio, un tel lieu étant jugé peu propice à la créativité.

\section{Titres connotatifs}

8 Ces titres de donnent pas d'« informations exactes » (Hoek $1981: 171$ ) sur le contenu du disque. Admettant une ou plusieurs connotations, ils doivent être interprétés sur la base de codes culturels partagés avec l'auditeur. Ces connotations peuvent porter sur différents objets :

\subsection{L'« ambiance » de la musique}

Jack Wilson, Easterly Winds ; The Three Sounds, Feelin' Good ; Stanley Turrentine, Blue Hour ; Ornette Coleman, Love Call. De toute évidence un titre comme « Easterly Winds »ne peut être pris littéralement : l'album ne consiste pas en enregistrements de vents, soufflant de l'est ou d'ailleurs, même si des enregistrements de ce type existent pour le plaisir des audiophiles et des écologistes. L'expression « Easterly Winds » est utilisée ici dans un sens connotatif («douceur»), pour décrire la musique pacifique de Wilson. De même, la tristesse que suggère «Blue » s'accorde culturellement aux morceaux sur tempo lent de 
Blue Hour, et l'idée de «bonheur » est inscrite dans les rythmes rapides de Feelin' Good. Quant à Love Call, ce titre joue sur les associations "primitivistes » que le jazz utilise fréquemment - associations justifiées dans ce cas par la violence et le caractère disruptif de la musique de Coleman.

\subsection{Le genre de la musique}

10 Freddie Roach, Mo' Greens Please ; Horace Parlan, Headin' South ; Jackie McLean, Destination Out. Alors que des titres comme Boogie-Woogie Classics and Afro-Cuban disent immédiatement à quel genre appartient l'album, Mo' Greens Please et Destination Out procèdent par association. En fait, ces titres renvoient à deux des tendances les plus représentées dans le catalogue Blue Note des années 1960. «Greens » désigne de manière métonymique le style «funk-soul » qu'annoncent plusieurs autres titres: George Braith, Soul Stream ; Lou Donaldson, Gravy Train ; Jimmy Smith, The Sermon ; Don Wilkerson, Preach Brother Preach. Quant au «Out» du titre de McLean, il désigne le «new », "free » jazz auquel d'autres titres renvoient de la même manière oblique : Joe Henderson, In 'n 'Out; Andrew Hill, Point of Departure ; Grachan Moncur, Evolution; Sam Rivers, A New Conception; et plus particulièrement Jackie McLean, It's Time, Let Freedom Ring, New Soil, One Step Beyond, et Right Now. Les connotations, bien sûr, sont parfois ambiguës. Le mot « Spring " (printemps) peut ainsi suggérer la fraîcheur, comme c'est le cas lorsqu'il est utilisé par des marques de parfum, de shampoing ou de déodorant. Mais il peut aussi signifier « rajeunissement », comme dans le titre du disque d'Anthony Williams Spring, un album qui contient des échantillons de la « nouvelle » musique des années 1960.

\subsection{Les relations de l'artiste à son album}

11 Art Blakey, Buhaina's Delight; Paul Chambers, The Whims of Chambers; Herbie Hancock, My Point of View ; Joe Henderson, Our Thing; Horace Parlan, Speakin' My Piece; McCoy Tyner, The Real McCoy. Les titres de ce type certifient que l'artiste prend plaisir à jouer la musique qu'il joue, voire même qu'il joue ce qu'il veut, sans compromettre les valeurs qui sont les siennes. A cet égard, des titres tels que Our Thing et The Real McCoy participent de l'idéologie de l'authenticité et de l'expressivité, souvent associée avec le jazz, idéologie dont il a déjà été question à propos des enregistrements « sur le vif. »

\section{Titres indexicaux}

12 Ces titres ne décrivent pas la musique de manière immédiate. Ils « indexent » simplement le disque sur lequel ils figurent, le distinguant des autres disques qu'on peut trouver dans les bacs des disquaires, dans les catalogues des éditeurs ou sur les rayons des étagères où l'acheteur les a finalement placés. Certes, tous les titres servent d'index. Mais certains d'entre eux n'exercent que cette fonction, dans la mesure où l'on voit mal comment ils pourraient caractériser la musique que contient l'album ou les musiciens qui l'interprètent. Les titres relevant de cette catégorie comprennent : 


\subsection{Une expression toute faite sortie de son emploi habituel}

13 Stanley Turrentine, Look Out; The Three Sounds, Good Deal; et tout particulièrement Wayne Shorter, Night Dreamer, Speak No Evil et The All Seeing Eye. Il va sans dire que certains de ces titres peuvent être considérés comme comportant une connotation: la musique de Donaldson établit une ambiance chaleureuse ( «sunny ») ; celle de Drew est atypique, elle appartient à un courant souterrain (« undercurrent »); et celle de Shorter a des aspects oniriques («night dreamer »). Mais d'autre titres semblent résister à toute récupération interprétative et fonctionnent strictement comme index. Les expressions Speak No Evil («ne dites pas de mal ») et The All Seeing Eye («l'œil qui voit tout»), par exemple, ne renvoient manifestement ni à la musique de Shorter, ni à quelque aspect connu de la personnalité du saxophoniste. Ces titres sont purement indexicaux, même si des critiques littéraires ingénieux pourraient sans doute en proposer des interprétations plausibles, comme ils le font dans leur lecture de poèmes particulièrement hermétiques.

\subsection{Un jeu de mots sur le nom (ou le surnom) du musician}

John Coltrane, Blue Train; Freddie Hubbard, Hub Cap ; Clifford Jordan, Cliff Craft ; Duke Jordan, Flight to Jordan ; Lee Morgan, Lee-Way, Delightfulee ; Louis Smith, Smithville. Certains de ces titres pourraient figurer dans la catégorie «relation de l'artiste à la musique ", dans la mesure où ils affirment que les interprètes font ce qu'ils veulent, indépendamment de toute pression commerciale (Lee-Way, Smithville). La fonction indexicale du titre est particulièrement manifeste dans le cas de Blue Train, la musique jouée par «Train» (surnom de Coltrane) n'étant pas «blue» dans le sens de «lente et mélancolique », comme elle l'est dans le Blue Hour de Turrentine. L'adjectif «blue » a probablement été choisi parce qu'il s'accordait phonétiquement avec « train », mieux par exemple que «orange» ou "yellow». La plupart des titres que l'on peut classer dans cette catégorie présentent d'ailleurs ce qu'Yvan Fonagy appelle des "figures de son » (1984 : 140), à savoir des combinaisons de jeux de mots et d'allitérations (Cliff Craft), des assonances (Smithville), et même les deux figures dans le même mot (Delightfulee).

\subsection{Une expression qui lie, parfois sous la forme d'un jeu de mots, le nom de l'artiste à la photographie ou au dessin qui figurent sur la pochette}

Donald Byrd, Byrd in Flight (la photographie montre un oiseau volant au-dessus de son nid) ; Sonny Clark, Dial $S$ for Sonny (la photographie montre Clark à côté d'un gros appareil de téléphone); Horace Silver, Horace Scope (un dessin représente les signes du zodiaque arrangés en cercle) ; Baby Face Willette, Face to Face (la photographie montre le visage de Willette, et l'expression " face to face » est aussi écrite à la fois de gauche à droite et de droite à gauche, les deux mots « face » se faisant littéralement face. 


\subsection{Une expression qui est liée à la photographie ou au dessin, mais qui ne joue pas sur le nom de l'artiste}

Lou Donaldson, Blues Walk (la photographie montre Donaldson se promenant) ; Gil Melle, Patterns in Jazz (la pochette, faite par Melle lui-même, présente une série de taches de couleur) ; Jimmy Smith, Crazy! Baby (la photographie montre une femme vêtue d'une blouse et d'un pantalon très collants, appuyée contre une Jaguar). Comme Hubcap ou Open Sesame, les titres qui constituent les catégories 3.3 et 3.4 sont aussi strictement indexicaux qu'il est possible de l'être. Certes, les images de hauteur dans Byrd in Flight et Lou Takes Off suggèrent que la qualité de la musique offerte dans ces disques est particulièrement «élevée ». Mais des métaphores aussi usées sont moins susceptibles d'attirer l'auditeur potentiel que le design lui-même, qui associe messages verbaux et iconographiques d'une manière esthétiquement séduisante. Si cet auditeur veut en savoir plus sur la musique elle-même et sur ses interprètes, il trouvera des informations (particulièrement riches dans le cas de Blue Note) au verso de la pochette du disque 33 tours, ou alors dans le livret qui accompagne le $\mathrm{CD}$.

Dans la mesure où ils nous donnent des renseignements sur la musique contenue dans les disques qu'ils identifient, les titres des albums Blue Note permettent de soulever une nouvelle fois la question de savoir si la musique réfère, et si elle réfère à quelque chose qui n'est pas elle-même. Comme Hayden White le demande en conclusion d'un colloque consacré aux relations entre musique et littérature : «La musique peut-elle produire des effets de sens semblables à ceux que produisent les poésies lyriques et narratives, la prose des fables et celle des romans, les discours didactique et conceptualisant? Peut-elle signifier, comme un tableau, une sculpture ou un monument architectural signifient? Permet-elle d'affirmer, de décrire, de référer, comme le langage le permet?» (1992 : 291). Les discussions sur ce sujet ont opposé, dans les termes du musicologue Leonard Meyer, les « référentialistes », pour qui « la signification musicale réside dans la relation entre un symbole ou un signe musical et l'objet extra-musical qu'il désigne » (1956:33), et les " absolutistes, " pour qui « un événement musical a une signification parce qu'il renvoie à un autre événement musical, dont il suscite l'attente » (1956: 35). Pour Umberto Eco, la musique a ainsi les caractéristiques d'un "système sémiotique sans niveau sémantique (ou plan du contenu)» (1976: 11); le signe graphique qui représente une note sur la portée, par exemple un sol, n'a pas de "profondeur sémantique », en ce sens qu'il ne fait que « dénoter une position dans un système de notes, une classe d'événements sonores qui ont pour interprétants des valeurs mathématiques et des mesures oscillographiques et spectographiques » (Eco $1976: 88$ ). De même, examinant les relations entre signifiant et signifié à l'intérieur du signe, Roman Jakobson distingue une "semiosis extroverse ", où le message possède une "composante référentielle» externe, d'une «semiosis introverse ", où le message "se signifie lui-même ». Les messages musicaux, selon lui, relèvent de la semiosis "introverse ", parce que la composante référentielle, dans ces messages, est « ou absente ou minime, même dans la musique dite à programme » (1971: 704-705). Quant à Igor Stravinsky, il affirme sans hésitations que la musique «n'a par essence pas le pouvoir d'exprimer quoi que ce soit: un sentiment, une attitude, un état psychologique, un phénomène naturel », ajoutant de la manière la plus « absolutiste » que «l'expression n'a jamais été une propriété immanente à la musique» (1971: 116). Certains sémioticiens et musicologues ont toutefois une vue moins restrictive du signe musical. Pour Jean-Jacques Nattiez, par exemple, les thèses de Jakobson et de Stravinsky 
ne sont pas exemptes de jugements normatifs ; les signes musicaux peuvent renvoyer au monde extérieur aussi bien qu'à d'autres signes musicaux, et le poids accordé aux deux variables tend à se modifier selon l'époque et les principes esthétiques (1975: 213). Charles Rosen use du même argument. Décrivant les théories qui accordent à la musique une fonction mimétique, il note que durant la période baroque "une fugue pouvait renvoyer à un sentiment ou à une émotion spécifiques" (1996: 70). Rosen souligne également que la musique, à l'époque romantique, avait le pouvoir de représenter la nature, par exemple que l'introduction de piano au Lied de Schubert « Der Lindenbaum » évoque par convention «des appels de cor et le bruit du vent dans les feuilles» (1996: 117). Les passages de ce type, selon Rosen, constituent des échantillons de l'« iconographie musicale des débuts du XIX e siècle ", iconographie qui, à l'époque, n'était pas considérée comme « imposée arbitrairement » $(1996: 135)$.

Les histoires du jazz et les études portant sur ses différents styles contiennent des discussions du même type. Plusieurs manuels, notamment Jazz Styles de Gridley et An Introduction to Jazz History de Megill et Demory, sont implicitement du côté des " absolutistes». Leurs auteurs caractérisent les mouvements successifs (New Orleans, swing, be-bop, etc.) en termes de traits formels, qu'ils renvoient à d'autres traits formels, selon des relations de "contraste, de symétrie/dissymétrie et de simplicité/ complication » - pour adopter la définition que donne Nattiez (1975: 138) de la position formaliste. Inversement, les critiques qui font les comptes-rendus de disques et de concerts semblent parfois présumer que la «bonne» musique doit exprimer quelque chose d'autre qu'elle-même, par exemple les sentiments de l'artiste. Ils se disent déçus lorsqu'Ella Fitzgerald se contente de "bien chanter» et ne montre pas, selon eux, suffisamment d'engagement existentiel dans ce qu'elle fait, ou lorsqu'il s'avère que Billie Holiday donnait ce que les spécialistes des gender studies appelleraient une "performance»: elle se contentait d'afficher, de «jouer " les émotions qu'elle était censée éprouver au plus profond d'elle-même.

Certaines histoires du jazz offrent toutefois les descriptions à la fois formalistes et culturelles dont des théoriciens comme Nattiez et Rosen se sont faits les avocats. L'anthropologue/musicologue Lucien Malson et le trompettiste Christian Bellest, dans leur introduction Le Jazz, expliquent ainsi que le style « jungle » de l'orchestre de Duke Ellington peut être défini par rapport à d'autres styles (la section de cuivres dans l'orchestre d'Ellington utilise davantage les sourdines que ne le font les sections de cuivres d'autres orchestres), mais ils ajoutent que, par rapport au domaine culturel noneuropéen, ce style semble aussi évoquer l'Afrique noire, dont Malson et Bellest ont la prudence de dire que la manière dont elle est représentée dans des morceaux aux titres exotiques tels que "Jungle Jamboree », "Echoes of the Jungle» et "Jungle Night in Harlem », est « imaginaire » et ne doit rien à des observations ethno-musicales (2007 : 52). Plus récemment, des critiques américains ont élargi la position référentialiste pour inclure les questions de race, de genre et d'orientation sexuelle qu'il est de règle de poser dans le domaine des cultural studies. Hazel Carby, par exemple, avance, dans le chapitre de Race Men qu'elle consacre à Miles Davis, que la musique de ce dernier concerne directement les problèmes de genre et de pouvoir. Adoptant la thèse de Susan McClary selon laquelle les morceaux instrumentaux sont des « textes culturels » qui doivent être interprétés en termes non seulement de leur "propriétés formelles » mais également de leur «contenu » (1998: 21), Carby affirme que la musique de Davis est révélatrice des « politiques sexuelles contradictoires » que le trompettiste a pratiquées et de «différents 
moments historiques » $(1998: 165)$ qu'il a traversés durant sa carrière. Plus précisément, Carby tente de démontrer que la musique de Davis a connu une importante évolution, qui l'a conduit, à la fin des années 1950, de la « phallocraticité » que manifestaient encore des albums tels que Milestones et 'Round Midnight, au « refus de résoudre la tension par un seul point culminant dans l'album » (Carby 1998 : 164) qui caractérise Kind of Blue. Pour Carby, en d'autres termes, les signes musicaux réfèrent à d'autres choses qu'eux-mêmes. En l'occurrence, la convention musicale «moment culminant " (climax) renvoie à l'attitude masculine, désignée comme "phallocentricité», un lien que Carby a aussi peu d'hésitations à établir que Rosen lorsqu'il entend « des appels de cor et le bruit du vent dans les feuilles» (1996: 117) dans les premières mesures du «Lindenbaum» de Schubert.

Les titres des disques Blue Note illustrent ces différentes théories de la «signification » de la musique, sans, bien sûr, que cela ait été l'intention des gens qui les ont conçus. Certains d'entre eux ne relèvent pas de la problématique de la référentialité. Un titre comme $A$ Night at Birdland, qui désigne le lieu où le disque a été enregistré, n'implique ainsi aucune conception de la référence musicale : il ne prétend pas que les morceaux qui constituent l'album dépeignent le Birdland, mais se borne à assurer que l'album contient, en partie du moins, la musique qui a été produite à cet endroit un certain soir. De même, Green Street ne proclame pas que la musique contenue dans le disque décrit une rue de couleur verte; comme je l'ai avancé précédemment, le titre indexe ici simplement l'album, qu'il distingue des autres enregistrements de Grant Green. D'autres titres, en revanche, illustrent la position "absolutiste» de Meyer. Par exemple, des expressions qui définissent un style, comme Dixieand Jubilee ou Afro-Cuban, impliquent que les événements musicaux ont une signification par rapport à d'autres événements musicaux dans la même catégorie sémiotique ; procédant par "équivalence " et "contraste " (Nattiez 1975), il décrivent ce qu'est la musique dans ces albums (" Dixieland », « Afro-Cuban »), et aussi, indirectement, ce qu'elle n'est pas (le «Dixieland» n'est pas vraiment du « New Orleans », et l'« Afro-Cuban » ne consttue ni du «Latin» ni du «Be-Bop»). Finalement, des titres connotatifs tels que Blue Hour et Feelin' Good exemplifient la position « référentialiste » de Meyer. En effet, ils impliquent que les signes musicaux peuvent désigner des objets non-musicaux, tout comme les signes linguistiques peuvent désigner des objets, des actions et des émotions non-linguistiques. Dans ces cas, comme je l'ai suggéré plus haut, ce sont les tempi de la musique qui renvoient conventionnellement à une certaine "ambiance ", que les adjectifs «blue » et " good " évoquent à l'aide d'une métaphore («blue ») et d'une qualification directe ( good »).

Les titres des albums d' " avant-garde » produits par Blue Note au début des années 1960 soulèvent de la manière la plus nette le problème de la référentialité de la musique. Pour poser la question de manière peut-être simpliste à propos de certains titres des disques du saxophoniste Jackie McLean : des expressions telles que Evolution, It's Time, Right Now, et Let Freedom Ring portent-elles sur la musique, décrivant le «nouveau » type de jazz que McLean et d'autres s'efforçaient de développer durant cette période ? Portent-elles sur le contexte, réclamant des changements dans la situation des Africains-Américains? Et, de façon plus importante pour mon propos, la musique que contiennent ces albums a-t-elle en elle-même une signification sociale? Dans son étude du jazz des années 1960, Frank Kofsky reprend la thèse, souvent formulée, selon laquelle les « révolutions » esthétique et sociale ne sauraient être séparées. Selon lui, les changements que le jazz avait connus durant cette période étaient autant de "réponses" à une "constellation massive de 
forces sociales et économiques ", notamment l'« augmentation du chômage dans la maind'œuvre noire peu qualifiée ", le désir des Africains-Américains "de ne plus être des citoyens de seconde classe", les «mouvements d'indépendance africaine» et la «croissance du nationalisme noir» (1998: 263). Mais Kofsky ne se demande pas si la musique de Coltrane et celle de Rivers peuvent référer à ces phénomènes, comme un roman, un poème et un tableau peuvent y référer. Quand il déclare que le titre It's Time signifie «l'heure de la libération est venue» (1998: 75), il interprète simplement l'expression à la lumière de certains aspects du contexte social ; il ne prétend pas que la musique que contient le disque décrit en fait ce contexte - ou qu'elle dépeint le développement d'un nationalisme noir et le chômage qui frappe les ghettos. Or, le problème que soulèvent des titres tels que Right Now et Let Freedom Ring est précisément de déterminer si la musique peut représenter ce type de contenu extra-musical. Dans It's Time, comme McLean l'explique lui-même dans les notes qui accompagnent ce disque, de nombreux signes musicaux inscrivent une libération, que ce soit dans le domaine des structures (trois des six morceaux sont basés sur le système modal, et non sur le schéma habituel AABA de trente-deux mesures), des rythmes (le 4/4 habituel est fréquemment abandonné), ou encore du traitement de l'instrument (McLean produit parfois des sons qui n'appartiennent pas au registre «normal» de l'instrument). Mais cette libération est spécifiquement musicale: McLean s'affranchit des conventions du hard-bop, de certaines d'entre elles du moins. Est-il légitime de l'étendre à d'autres systèmes sémiotiques? Avons-nous le code qui nous dirait comment effectuer ce transfert? Dans le cas présent, savons-nous comment attribuer un signifié à un signifiant musical tel que le système modal ou les sons hors registre que Mclean tire de son instrument?

Il est difficile d'offrir des réponses nouvelles et convaincantes à des questions qui divisent toujours la communauté des musicologues. Comme Nattiez, Rosen et Carby le soutiennent, cependant, il n'y a pas de raison à ce que les positions «absolutiste » et « référentialiste» s'excluent l'une l'autre. En l'occurrence, si nous admettons que les titres Blue Note constituent (entre autres choses) ce que Peter Rabinowitz appelle des " instructions d'écoute ", à savoir des énoncés verbaux qui fournissent « une structure à travers laquelle sera traitée l'expérience des sons » (1992: 41-42), des expressions telles que It's Time, Right Now et Let Freedom Ring peuvent être considérées comme des invitations à procéder - dans la terminologie de Jakobson - à des semiosis à la fois introverses et extroverses. Pour le dire autrement, ces expressions engagent l'auditeur à traiter la musique qu'il entend d'une manière " absolutiste » - à la situer par rapport aux autres types de jazz pratiqués à l'époque ou antérieurement. Mais elles l'incitent également à exercer une écoute "référentialiste » - à traiter cette même musique comme ayant une dimension sociale et politique. Certes, les invitations qu'adressent les titres de McLean ne renvoient pas aussi explicitement à des événements connus que le font Fables of Faubus de Charlie Mingus, qui nomme le gouverneur de l'Arkansas qui avait bloqué les mesures d'intégration scolaire voulues par Washington, ou Attica Blues d'Archie Shepp, qui nomme tout aussi expressément la prison dans laquelle une révolte de détenus (en grande majorité noirs) avait été brutalement réprimée. De plus, à supposer que la musique de McLean ait un programme, les auteurs des notes qui accompagnent les albums dont il a été question (soit McLean lui-même et le critique Nat Hentoff, connu pour ses opinions « de gauche ») n'ont pas fait ce que font leurs collègues dans le domaine de la musique classique lorsqu'ils présentent, par exemple, le Don Juan, le Till Eulenspiegel ou la Symphonie alpine de Richard Strauss : ils n'ont pas expliqué le programme annoncé par les titres, ce qui aiderait l'auditeur à suivre l'histoire ou à comprendre l'argument. 
Dans le cas de It's Time et d'autres albums Blue Note datant du début des années 1960, l'auditeur qui s'applique à une écoute "référentialiste» peut donc adopter deux stratégies interprétatives qui ne sont d'ailleurs pas incompatibles : il peut voir dans cette musique des appels à une prise de conscience sociale et politique, ou il peut chercher des équivalences ponctuelles entre certains signes musicaux et le monde extérieur. Les sons hors registre que produit McLean pourraient ainsi être compris comme des gestes de colère et de révolte, dont la fonction est de traduire, sur un autre plan sémiotique, la colère et la révolte qu'inspirent les inégalités raciales. Les titres des albums Blue Note procurent-ils d'autres "grilles interprétatives", qui permettraient à l'auditeur d'« attribuer » à ce qu'il entend une signification plus spécifique (Rabinowitz 1992 : 42) ? Probablement pas. Mais, dans la mesure où ils nous aident à poser une fois encore le problème de la référentialité de la musique, ces mêmes titres contribuent à un débat qui reste d'actualité en sémiotique aussi bien qu'en musicologie.

\section{BIBLIOGRAPHIE}

\section{Références}

(1987), « The Complete Blue Note Book. Tribute to Alfred Lion », in Jazz Critique, $\mathrm{N}^{\circ} 2$.

CARBY, Hazel (1998), Race Men, Cambridge (MA), Harvard University Press.

CUSCUNA, Michel, et Michael RUPPLI (1988), The Blue Note Label. A Discography, Greenport (CT), Greenwood Press.

DERRIDA, Jacques (1981), « Title (to be specified) », Sub-Stance, № 31, p. 5-22.

ECO, Umberto (1976), A Theory of Semiotics, Bloomington, Indiana University Press.

FONAGY, Yvan (1984), « La poésie des titres ", in Semiosis. Semiotics and the History of Culture, Morris Halle, Krystyna Pomorska, Ladislav Matejka \& Boris Uspenskij (dir.), Ann Arbor, Michigan Slavic Publications, p. 139-156.

GENETTE, Gérard (1987), Seuils. Paris, Seuil.

HOEK, Leo. H. (1981), La Marque du titre. Dispositifs sémiotiques d'une pratique textuelle, La Haye, Mouton.

JAKOBSON, Roman (1971), « Language in Relation to Other Communication Systems », Selected Writings, vol. II, La Haye, Mouton, p. 697-708.

KOFSKY, Frank, (1998), John Coltrane and the Jazz Revolution of the 1960s, New York, Pathfinder.

MALSON, Lucien \& Christian BELLEST (2007), Le Jazz, Paris, P.U.F.,coll. « Que sais-je? ».

MCCLARY, Susan (1997), " The Impromptu that Trod on the Loaf: or, How Music tells Stories ", Narrative, vol. 5, $\mathrm{N}^{\circ} 1$, p. 20-35.

MEYER, Leonard (1956), Emotion and Meaning in Music, Chicago, University of Chicago Press. 
NATTIEZ, Jean-Jacques (1975), Fondements d'une sémiologie de la musique, Paris, Union Générale d'Editions.

RABINOWITZ, Peter, (1992), «Chord and Discord. Listening Through the Written Word », Music and Text. Critical Inquiries, Steven Paul Scher (dir.), Cambridge, Cambridge University Press, p. 38-56.

ROSEN, Charles (1996), The Romantic Generation, Londres, Harper Collins.

STRAVINSKI, Igor (1971), Chroniques de ma vie, Paris, Médiations Gonthier.

WHITE, Hayden (1992), « Form, reference and Ideology in Musical Discourse », in Music and Text. Critical Inquiries, Steven Paul Scher (dir.), Cambridge, Cambridge Uviversity Press, p. 288-319.

\section{Titres des albums cités}

Les dates sont celles des enregistrements, telles qu'elles sont données dans la discographie de Cuscuna et Ruppli (1988). Cet ouvrage ne dit pas quand le disque est sorti, et cette information ne figure en général pas sur la pochette du disque.

AMMONS, Albert. Boogie-Woogie Classics. Blue Note, 1939.

BLAKEY, Art. A Night at Birdland with Art Blakey Quintet. Blue Note, 1954.

BLAKEY, Art. Buhaina's Delight. Blue Note, 1961.

BRAITH, George. Soul Stream. Blue Note, 1963.

BROWN, Clifford. Memorial Album. Blue Note, 1953.

BURELL, Kenny. Introducing Kenny Burrell. Blue Note, 1956.

BYRD, Donald. At the Half Note Cafe. Blue Note, 1960.

BYRD, Donald. Byrd in Flight. Blue Note, 1960.

BYRD, Donald. Free Form. Blue Note, 1961.

CHAMBERS, Paul. Whims of Chambers. Blue Note, 1956.

CLARK, Sonny. Dial S for Sonny. Blue Note, 1957.

COLEMAN, Ornette. Love Call. Blue Note, 1968.

COLTRANE, John. Blue Train. Blue Note, 1957.

DONALDSON, Lou. Blues Walk. Blue Note, 1958.

DONALDSON, Gravy Train. Blue Note, 1961.

DONALDSON, Lou Donaldson Sextet. New York: Blue Note, 1954.

DONALDSON, Lou Takes Off. Blue Note, 1957.

DONALDSON, Sunny Side Up. Blue Note, 1960.

DORHAM, Kenny. Afro-Cuban. Blue Note, 1955.

DORHAM, Kenny.'Round Midnight at the Cafe Bohemia. Blue Note, 1956.

DREW, Kenny. Undercurrent. Blue Note, 1960.

FARLOW, Tal. Tal Farlow Quartet. Blue Note, 1954.

GREEN, Grant. Green Street. Blue Note, 1961. 
GREEN, Grant. The Latin Bit. Blue Note, 1962.

HANCOCK, Herbie. My Point of View. Blue Note, 1963.

HENDERSON, Joe. In 'n Out. Blue Note, 1964.

HENDERSON, Joe. Our Thing. Blue Note, 1963.

HILL, Andrew. Andrew. Blue Note, 1964.

HILL, Andrew. Point of Departure. Blue Note, 1964.

HIPP, Jutta. Jutta Hipp at the Hickory House. Blue Note, 1956.

HODES, Art. Dixieland Jubilee. Blue Note, 1944.

HUBBARD, Freddie. Hub Cap. Blue Note, 1961.

HUBBARD, Freddie. Open Sesame. Blue Note, 1960.

JOHNSON, J.J. The Eminent J.J. Johnson. Blue Note, 1953, Jordan, Clifford. Cliff Craft. Blue Note, 1957.

JORDAN, Clifford, and GILMORE, Don. Blowing In From Chicago. Blue Note, 1957.

JORDAN, Duke. Flight from Jordan. Blue Note, 1960.

LEWIS, George. Echoes of New-Orleans. Blue Note, 1943.

McLEAN, Jackie. Destination Out. Blue Note, 1963.

McLEAN, Jackie. It's Time. Blue Note, 1964.

McLEAN, Jackie Let Freedom Ring. Blue Note, 1962.

McLEAN, Jackie New Soil. Blue Note, 1959.

McLEAN, Jackie One Step Beyond. Blue Note, 1963.

McLEAN, Jackie Right Now. Blue Note, 1965.

MELLE, Gil. Patterns in Jazz. Blue Note, 1956.

MOBLEY, Hank. Hank Mobley and His All-Stars. Blue Note, 1957.

MONCUR, Grachan. Evolution. Blue Note, 1963.

MORGAN, Lee. Delightfulee. Blue Note, 1966.

MORGAN, Lee. Lee-Way. Blue Note, 1960.

NAVARRO, Fats. The Fabulous Fats Navarro. Blue Note, 1947.

PARLAN, Horace. Speakin' My Piece. Blue Note, 1960.

POWELL, Bud. Bud! Blue Note, 1957.

QUEBEC, Ike. Heavy Soul. Blue Note, 1961.

REDD, Freddie. The Music from the Connection. Blue Note, 1960.

RIVERS, Sam. A New Conception. Blue Note, 1966.

ROACH, Freddie. Mo' Grease Please. Blue Note, 1963.

ROLLINS, Sonny. A Night at the Village Vanguard. Blue Note, 1957.

ROLLINS, Sonny. Sonny Rollins. Blue Note, 1956.

ROUSE, Charlie. Bossa Nova Bacchanal. Blue Note, 1962. 
SHORTER, Wayne. Night Dreamer. Blue Note, 1964.

SHORTER, Wayne. Speak No Evil. Blue Note, 1964.

SHORTER, Wayne. The All Seeing Eye. Blue Note, 1965.

SILVER, Horace. Horace-Scope. Blue Note, 1960.

SILVER, Horace. Six Pieces of Silver. Blue Note, 1956.

SMITH, Jimmie. Crazy! Baby. Blue Note, 1960.

SMITH, Jimmie. Groovin' at Small's Paradise. Blue Note, 1957.

SMITH, Jimmie. Plays Fats Waller. Blue Note, 1962.

SMITH, Jimmie. The Sermon. Blue Note, 1957.

SMITH, Louis. Smithville. Blue Note, 1958. The Three Sounds. Good Deal. Blue Note, 1959.

SMITH, Louis. Feelin' Good. Blue Note, 1960.

TURRENTINE, Stanley. Blue Hour. Blue Note, 1960.

TURRENTINE, Stanley. Look Out. Blue Note, 1960.

TURRENTINE, Stanley. Up at Minton's. Blue Note, 1961.

TYNER, McCoy. The Real McCoy. Blue Note, 1967.

WILKERSON, Don. Preach Brother. Blue Note, 1962.

WILLETTE, « Baby Face », Face to Face. Blue Note, 1961.

WILLIAMS, Anthony. Spring. Blue Note, 1965.

WILSON, Jack. Easterly Winds. Blue Note, 1967.

\section{ANNEXES}

Philippe Carrard a enseigné à l'Université de Pennsylvanie, à l'Université de Californie à Santa Barbara et à l'Université du Vermont. Il est actuellement « Visiting Scholar » dans le programme de littérature comparée à Dartmouth College (New Hampshire, Etats-Unis). Il est l'auteur de Malraux ou le récit hybride, Poétique de la Nouvelle Histoire et Nous avons combattu pour Hitler, de même que de nombreux articles sur les conventions d'écriture dans le discours non-fictionnel.

\section{RÉSUMÉS}

Les titres qui figurent sur la pochette des disques de la marque Blue Note peuvent dénoter le contenu musical de l'album (Boogie-Woogie Classics), le connoter (Blue Hour) ou fonctionner strictement comme index (Speak No Evil). Quelle que soit leur valeur précise, ces titres permettent de revisiter un problème maintes fois soulevé par les musicologues et les sémioticiens: celui de savoir si la musique «réfère », et à quoi. Dans le cas des disques Blue Note, la question se pose d'une manière particulièrement aiguë à propos de certains enregistrements des années 1960, dont les titres (It's Time, Right Now, Let Freedom Ring, etc.) peuvent référer aux structures du jazz 
ou à la situation des noirs dans la société américaine, qu'il s'agit tous deux de transformer dans le sens d'une liberté accrue.

The titles on the jackets of the records of the label Blue Note can denote the musical content of the album (Boogie-Woogie Classics), connote it (Blue Hour), or function strictly as indexes (Speak No Evil). Whatever their exact value might be, those titles make it possible to revisit a problem frequently raised in semiotics and musicology: the problem of knowing whether music " refers ", and to what exactly. The question is especially acute in the case of some of the records released by Blue Note in the1960s, whose titles (It's Time, Right Now, Let Freedom Ring) may refer to musical structures or to the situation of African-Americans in American sociey, which have both to be changed in the sense of more freedom.

\section{INDEX}

Mots-clés : absolutiste, connotatif, dénotatif, indexical, instructions d'écoute, paratexte, pochette, poétique, référentialiste, titre

\section{AUTEUR}

\section{PHILIPPE CARRARD}

Université du Vermont et Dartmouth College 